05

\title{
Влияние микротрещин на коэффициент Пуассона при пластическом деформировании аустенитной стали
}

\author{
(C) С.В. Кириков, В.В. Мишакин, В.А. Клюшников
}

Институт проблем машиностроения РАН - филиал Федерального исследовательского центра ФГБНУ

„Институт прикладной физики РАН“,

603024 Нижний Новгород, Россия

e-mail: imndt31@mts-nn.ru

Поступило в Редакцию 15 октября 2021 г.

В окончательной редакции 7 декабря 2021 г.

Принято к публикации 8 декабря 2021 г.

Исследовано влияние накопления поврежденности на коэффициент Пуассона, измеренного эхоимпульсным акустическим методом, при пластическом деформировании стали $12 \mathrm{X} 18 \mathrm{H} 10 \mathrm{~T}$. На основе полученных экспериментальных данных рассчитаны парциальные вклады в изменение коэффициента Пуассона вследствие накопления поврежденности и выделения фазы деформационного мартенсита. Методом компьютерного моделирования проведен анализ характеристик стабильных трещин, формирующихся вблизи частиц деформационного мартенсита при малых степенях пластической деформации. Построена теоретическая зависимость изменения коэффициента Пуассона вследствие образования трещин при пластическом деформировании. Получено хорошее совпадение экспериментальных данных и теоретических расчетов.

Ключевые слова: пластическое деформирование, аустенитная сталь, коэффициент Пуассона, мартенситное превращение, дислокация, мезодефект, метод конфигурационной силы.

DOI: 10.21883/JTF.2022.03.52135.277-21

\section{Введение}

Известно, что пластическое деформирование хромоникелевых сталей с низкой энергией дефектов упаковки сопровождается образованием фазы деформационного мартенсита из исходной фазы аустенита. Выделение мартенситной фазы, имеющей более высокие прочностные свойства по сравнению с фазой аустенита, приводит к изменению электромагнитных, упругих и акустических свойств всего материала, а также оказывает влияние на процесс накопления поврежденности в более мягком аустените [1-5]. В [6] показано, что плотность микротрещин имеет высокую корреляцию с объемной долей деформационного мартенсита.

В работе [1] получено, что коэффициент Пуассона, определяемый с помощью акустических измерений, можно использовать в качестве диагностического структурно-чувствительного параметра состояния аустенитной стали при усталостном разрушении. Коэффициент Пуассона $v$ выражается через времена или скорости упругих волн следующим образом:

$$
v=\frac{\tau_{\tau}^{2}-2 \tau_{l}^{2}}{2\left(\tau_{\tau}^{2}-\tau_{l}^{2}\right)}=\frac{V_{l}^{2}-2 V_{\tau}^{2}}{2\left(V_{l}^{2}-V_{\tau}^{2}\right)}
$$

где $V_{\tau}, V_{l}, t_{\tau}, t_{l}-$ скорости и времена распространения поперечных и продольных упругих волн соответственно.

В процессе деформирования на коэффициент Пуассона $v$ оказывают влияние в основном два фактора накопление поврежденности и образование фазы деформационного мартенсита:

$$
\Delta v=\Delta v_{\psi}+\Delta v_{\Phi}
$$

где $\Delta v_{\psi}, \Delta v_{\Phi}$ - изменения коэффициента Пуассона вследствие накопления поврежденности и выделения фазы деформационного мартенсита соответственно.

Согласно теоретическим моделям, приведенным в работах [7-10], образование и развитие трещин приводит к уменьшению модулей упругости и величины $v$, образование мартенсита к увеличению $v$. Изменение коэффициента Пуассона $\Delta v_{\psi}$ вследствие накопления поврежденности $\psi$ в работе [10] определяется следующим образом:

$$
\Delta v_{\psi}=-\frac{3\left(1-v_{0}^{2}\right)\left(5 v_{0}-1\right)}{2\left(7-5 v_{0}\right)} \psi,
$$

где $v_{0}$ - коэффициент Пуассона неповрежденного материала, $\psi=n_{\mathrm{cr}} \bar{l}^{3}, n_{\mathrm{cr}}-$ концентрация трещин, $l$ - средняя длина трещин.

Основываясь на имеющихся электронно-микроскопических исследованиях [11], можно предположить, что существуют три характерных стадии накопления повреждений, связанных с образованием фазы деформационного мартенсита в аустенитных сталях при активной пластической деформации:

- - на первой стадии происходит увеличение объемной доли частиц мартенсита и накопление плотности микротрещин вблизи этих частиц;

- - вторая стадия характеризуется значительным увеличением плотности и длины микротрещин, образованных на частицах деформационного мартенсита;

- - на третьей стадии происходит потеря устойчивости микротрещины и превращение ее в магистральную трещину. 
На первой и второй стадиях происходит увеличение плотности стабильных трещин в полях внутренних напряжений от мезодефектов [12-14], формирующихся на частицах деформационного мартенсита в процессе пластического деформирования. В настоящее время остается открытым вопрос о модельном представлении процесса накопления микроповрежденности (трещин), связанного с образованием частиц мартенсита. Построение такой модели и сравнение результатов теоретических расчетов с результатами экспериментальных исследований даст более полную картину разрушения метастабильной стали аустенитного класса, подвергаемой деформированию.

В настоящей работе на основе экспериментальных данных проведено численное моделирование характеристик стабильных микротрещин, формирующихся на частицах деформационного мартенсита в процессе пластического деформирования, и исследовано их влияние на коэффициент Пуассона аустенитной хромоникелевой стали 12Х18Н10T.

\section{1. Методика проведения эксперимента}

Исследования процесса пластического деформирования стали 12Х18Н10Т проводились методами ультразвукового и вихретокового вида контроля. Химический состав исследуемой стали (mass. \%): С 0.02, Si 0.43, Mn 0.74, Cr 17.76, Ni 9.16, Ti 0.32, S 0.002, P 0.033, $\mathrm{Cu} 0.23$, основа Fe.

Проводилось одноосное растяжение со скоростью деформации $10^{-4} \mathrm{~s}^{-1}$ при комнатной температуре. Длина рабочей части плоского образца составляла $100 \mathrm{~mm}$, ширина $-20 \mathrm{~mm}$, толщина $-6 \mathrm{~mm}$. Испытания проводились на электромеханической универсальной разрывной машине Tinius Olsen H100KU. Деформирование осуществлялось поэтапно, ультразвуковые и вихретоковые исследования выполнялись до первого этапа (в исходном состоянии) и после каждого этапа нагружения вплоть до образования шейки.

Время распространения упругих волн измерялось с помощью ультразвукового эхо-метода. Для возбуждения сдвиговых и продольных волн применялись прямые пьезоэлектрические преобразователи соответственно V156 и V110 производства Olympus с диаметром рабочей пластины $6 \mathrm{~mm}$ и несущей частотой $5 \mathrm{MHz}$. Продольные и поперечные волны распространялись перпендикулярно оси нагружения. Поляризация поперечных волн была направлена как вдоль оси нагружения, так и поперек.

В качестве генератора электрических импульсов использовался ультразвуковой дефектоскоп А1212 MACTEP. Цифровой осциллограф ADCLab был использован для записи на персональный компьютер амплитудно-временны́х диаграмм эхо-импульсов, поступающих с пьезопреобразователей на персональный компьютер. Частота дискретизации - $1 \mathrm{GHz}$, временно́е разрешение - $1 \mathrm{~ns}$. В результате обработки данных были получены времена распространения упругих волн $t_{\tau}$ и $t_{l}$ после каждого этапа нагружения. При расчете $t_{\tau}$ использовалось усредненное значение времен распространения поперечных волн, поляризованных вдоль и поперек оси нагружения.

Погрешность измерения времени распространения составляла $2-3 \mathrm{~ns}$, определения коэффициента Пуассона $-7 \cdot 10^{-4}$.

Измерение объемной доли фазы деформационного мартенсита $\Phi$ проводили с помощью многофункционального вихретокового прибора „МВП-2М“ после каждого этапа нагружения. Прибор был предварительно откалиброван на образцах с известным содержанием мартенситной фазы. Относительная погрешность измерения не превышала 5\%.

\section{2. Результаты экспериментальных исследований}

Вихретоковые исследования показали изменение электромагнитных характеристик стали 12Х18Н10Т в процессе пластического деформирования. Зависимость объемной доли фазы деформационного мартенсита $\Phi$ от величины пластической деформации $\varepsilon_{p l}$ имеет сигмоидальную форму (рис. $1, a$ ), математическое описание которой было первоначально приведено в работе [15].

В результате акустических исследований были получены времена распространения сдвиговых и продольных упругих волн и рассчитан коэффициент Пуассона по формуле (1) (рис. $1, b)$. Получено, что на начальном участке кривой $v\left(\varepsilon_{p l}\right)$ скорость изменения коэффициента Пуассона $\Delta v / \Delta \varepsilon_{p l}$ максимальная. Увеличение коэффициента Пуассона связано с изменением модулей упругости всего материала, на которые оказывает влияние образование частиц деформационного мартенсита. На участке развитой пластической деформации дефекты дислокационного типа индуцируют появление вблизи этих частиц неоднородных полей упругих напряжений, что провоцирует зарождение и распространение микротрещин. Развитие поврежденности приводит к уменьшению величины $\Delta v$, выражение (3).

Предполагается, что на начальных участках деформирования зависимость $\Delta v(\Phi)$ имеет линейный характер (рис. $1, c)$, и коэффициент Пуассона определяется только выделением магнитной фазы, тогда изменение $\Delta v_{\Phi}$, входящее в выражение (2), имеет вид

$$
\Delta v_{\Phi}=k_{\Phi} \Delta \Phi,
$$

где $k_{\Phi}=0.0055$ (в формуле (4) объемная доля выражена в долях, на рисунках в процентах).

Используя экспериментальные данные изменения коэффициента Пуассона вследствие накопления поврежденности $\Delta v_{\psi}^{\text {(ехр) }}$ можно определить из выражений (2) и (4) как

$$
\Delta v_{\psi}^{(\exp )}=\Delta v-k_{\Phi} \Delta \Phi .
$$



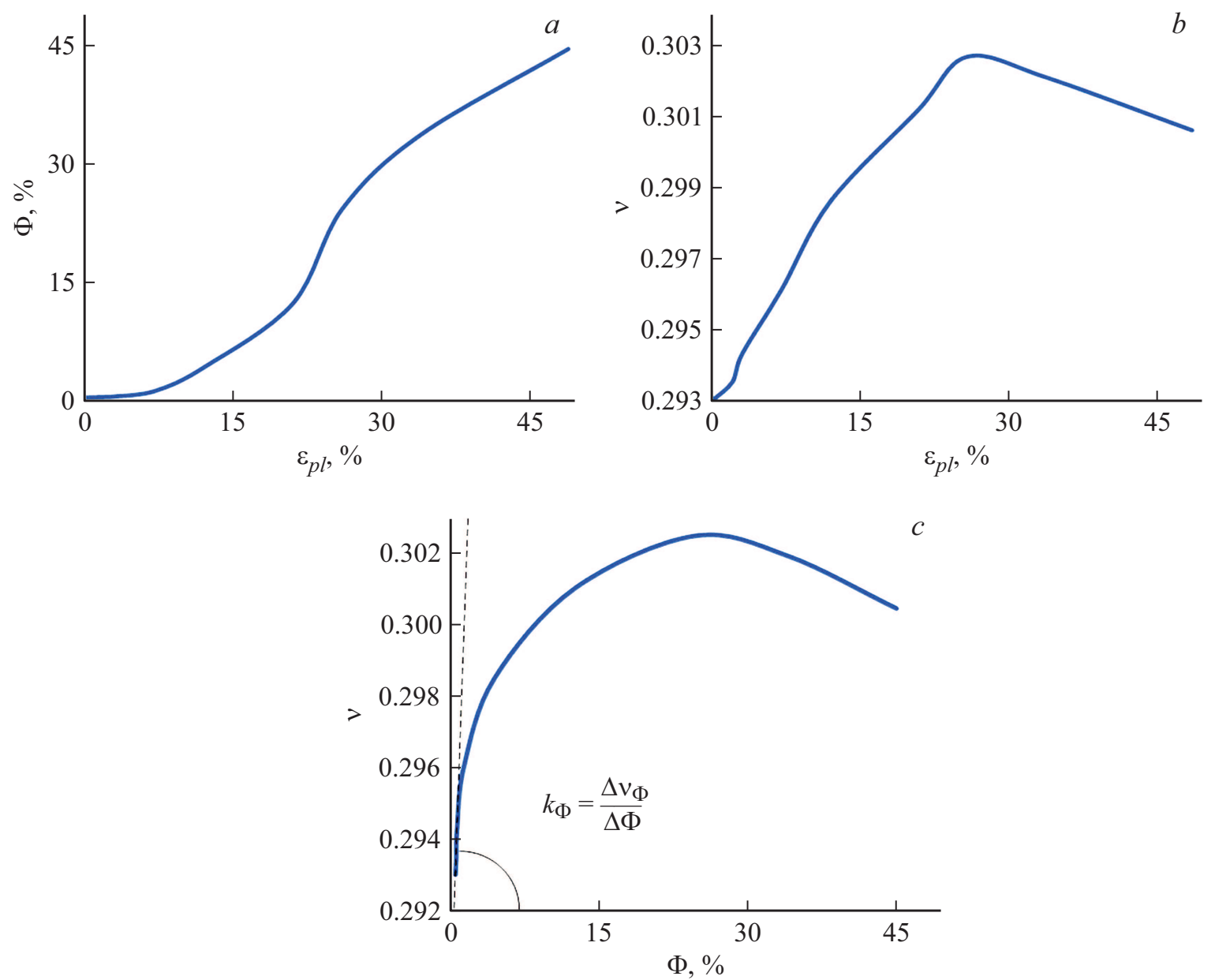

Рис. 1. Зависимости объемной доли фазы мартенсита $\Phi(a)$ и коэффициента Пуассона $(b)$ от величины пластической деформации $\varepsilon_{p l}$, связь $v(\Phi)(c)$.

На рис. 2 показано изменение коэффициента Пуассона $v$ и его составляющих $v_{\psi}^{(\exp )}$ и $v_{\Phi}$.

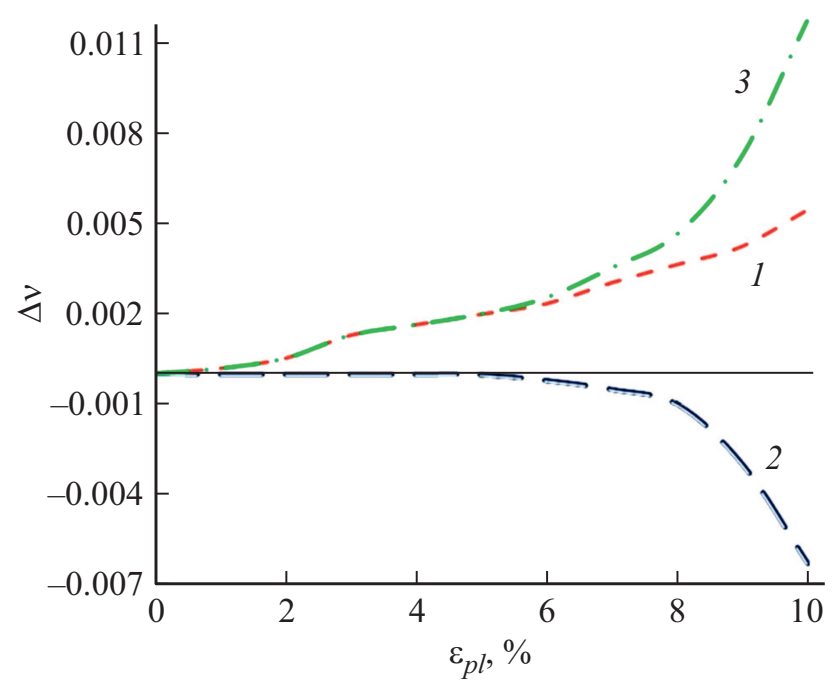

Рис. 2. Изменение коэффициента Пуассона $v(1)$ и его составляющих $v_{\psi}^{(\exp )}(2), v_{\Phi}(3)$ на ранних стадиях пластического нагружения.
Получено, что поврежденность не оказывает влияние на коэффициент Пуассона $v_{\psi}$ при деформациях $\varepsilon_{p l}$, не превышающих 5\%. При дальнейшем деформировании фаза мартенсита начинает выделяться более интенсивно (рис. $1, a)$, что сказывается на составляющей $\Delta v_{\Phi}$.

\section{3. Моделирование}

Проведем теоретическое исследование изменения коэффициента Пуассона вследствие накопления поврежденности (плотности трещин). Поставленную задачу будем решать в $2 D$-приближении (в приближении плоской деформации). Аустенитную матрицу представим в виде бесконечной упругоизотропной сплошной среды, которая характеризуется модулем сдвига $G$ и коэффициентом Пуассона $v$. Матрица содержит изотропные частицы второй фазы, имеющие форму прямоугольников, одинаковых размеров с длинами сторон, равными $2 a_{1}$ и $2 a_{2}\left(a_{1} \gg a_{2}\right)$. Пусть распределение ориентаций данных частиц по углу $\Omega \in[0,2 \pi)$ является изотропным (рис. $3, a)$. С учетом симметрии схемы деформирования 


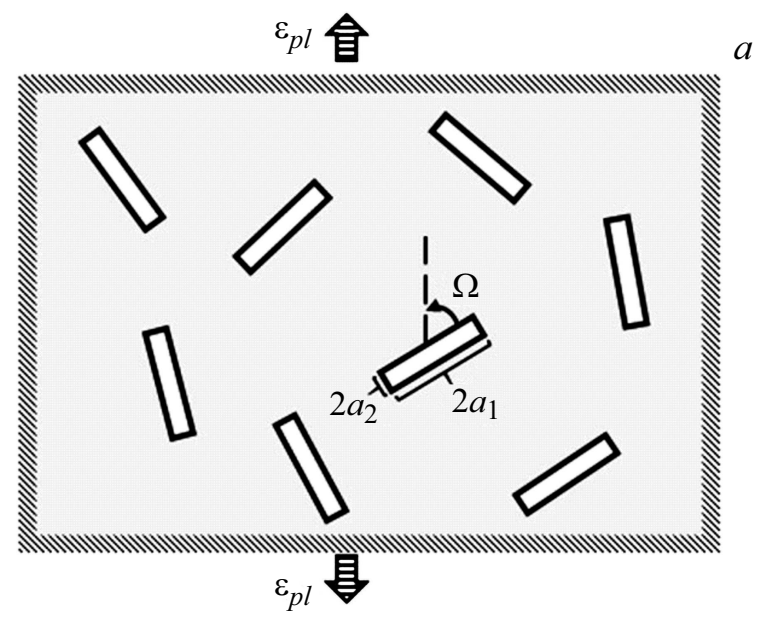

Рис. 3. Схематичное представление аустенитной матрицы, содержащей пластинчатые частицы мартенсита ( $a$ ), и системы координат, связанной с частицей мартенсита $(b)$.

и формы частицы угол $\Omega$ достаточно варьировать в диапазоне $[0, \pi / 2]$.

Так как значение предела текучести фазы деформационного мартенсита значительно больше, чем значение предела текучести матрицы аустенита [16], в рассматриваемой модели будем считать, что прямоугольная частица пластически недеформируема.

Декартову систему координат $O x y z$ и ассоциированный с ней базис $\left(\mathbf{e}_{1}, \mathbf{e}_{2}, \mathbf{e}_{3}\right)$ свяжем с частицей (рис. $\left.3, b\right)$. Концентрация частиц $d n_{m}\left(\Omega, \varepsilon_{p l}\right)$ мартенсита, ориентированных в интервале $[\Omega, \Omega+d \Omega]$, при деформации аустенитной матрицы $\varepsilon_{p l}$ для заданной геометрии определяется как

$$
d n_{m}\left(\Omega, \varepsilon_{p l}\right)=\frac{1 \mu \mathrm{m}^{3} \Phi\left(\varepsilon_{p l}\right)}{1 \mu \mathrm{m} S_{m}} \frac{d \Omega}{\pi / 2},
$$

где $\Phi\left(\varepsilon_{p l}\right)$ - объемная доля частиц мартенсита при величине пластической деформации $\varepsilon_{p l}, S_{m}$ - площадь частицы мартенсита, $d \Omega /(\pi / 2)$ - доля частиц, ориентация которых лежит в интервале $[\Omega, \Omega+d \Omega]$.

В процессе пластического деформирования аустенитной матрицы на границах мартенситных частиц и в их окрестностях накапливается избыточная плотность дислокаций, которые создают неоднородные поля внутренних напряжений. В общем случае распределение дислокационной плотности (распределение пластических деформаций) вблизи границы частицы имеет достаточно сложную неоднородную структуру, однако при малых степенях деформирования можно условно считать, что вся дислокационная плотность будет распределена по границе включения примерно однородно. Несмотря на то, что данное допущение является довольно грубым приближением реального распределения пластических деформаций вблизи недеформируемого включения, оно позволяет задавать простую и в то же время согласованную с пластической деформацией систему внутренних источников напряжений и достаточно легко описывать структуру полей напряжений вблизи частицы.
Плотность дислокаций ориентационного несоответствия, наведенных на границу частицы, характеризуется скачком пластической деформации $\left[\varepsilon_{\mathrm{pl}}\right]$ на этой границе, который зависит от величины $\varepsilon_{p l}$ и от ориентации частицы $\Omega$ :

$$
\left[\boldsymbol{\varepsilon}_{\mathbf{p l}}\right]=\varepsilon_{p l}\left(\mathbf{n}(\Omega) \otimes \mathbf{n}(\Omega)-\mathbf{n}_{\perp}(\Omega) \otimes \mathbf{n}_{\perp}(\Omega)\right), \quad \varepsilon_{p l}>0 .
$$

Для удобства дальнейших расчетов представим поля напряжений, связанные с собственной однородной дилатацией частицы $\varepsilon_{0}$, как поля, генерируемые виртуальными дислокациями, распределенными по границе включения $[17,18]$. Отметим, что из-за наличия собственной дилатации мартенситной частицы в ней создаются достаточно большие сжимающие напряжения. Тензор плотности вектора Бюргерса В [19] дислокаций на границе частицы мартенсита с нормалью $\mathbf{N}$ (внутренней по отношению к частице) определяется как [20]:

$$
\mathbf{B}=-\mathbf{N} \times\left(\left[\varepsilon_{\mathbf{p l}}\right]-\left[\boldsymbol{\varepsilon}_{\mathbf{0}}\right]\right),
$$

где $\left[\boldsymbol{\varepsilon}_{\mathbf{0}}\right]$ имеет вид

$$
\left[\boldsymbol{\varepsilon}_{0}\right]=\varepsilon_{0}\left(\mathbf{e}_{1} \otimes \mathbf{e}_{1}+\mathbf{e}_{2} \otimes \mathbf{e}_{2}\right), \varepsilon_{0}>0 .
$$

Полученное распределение виртуальных дислокаций с точки зрения полей упругих напряжений удобно характеризовать в терминах мезодефектов простой структуры: диполей клиновых дисклинаций мощности $\mathbf{w}_{\mathbf{N}}$ и планарных сдвиговых мезодефектов мощности $\mathbf{w} \boldsymbol{\tau}$ [21]. Выражения для мощностей мезодефектов $\mathbf{w}_{\mathbf{N}}$ и $\mathbf{w} \boldsymbol{\tau}$ на границе с нормалью $\mathbf{N}$ имеют вид:

$$
\mathbf{w}_{\mathbf{N}}=\left(\mathbf{e}_{3} \cdot \mathbf{B} \cdot \mathbf{N}\right) \mathbf{N} ; \quad \mathbf{w}_{\boldsymbol{\tau}}=\left(\mathbf{e}_{3} \cdot \mathbf{B} \cdot \boldsymbol{\tau}\right) \boldsymbol{\tau},
$$

где $\boldsymbol{\tau}=\mathbf{N} \times \mathbf{e}_{\mathbf{3}}-$ единичный вектор, направленный вдоль границы частицы. Выражения для расчета компонент тензора полей упругих напряжений для указанных мезодефектов приведены в Приложении [22,23]. 
Далее проанализируем характеристики стабильных трещин, зарождающихся вблизи ротационно-сдвиговых мезодефектов, формирующихся в процессе пластического деформирования аустенита на границе мартенситного включения. Отметим, что используемый в настоящей работе подход связан с представлением внутренних полей напряжений от частицы с помощью эквивалентной системы виртуальных мезодефектов и дальнейшим исследованием зарождения и распространения микротрещин широко используется в настоящее время для построения моделей разрушения [24-27]. Будем считать, что микротрещина формируется на отрицательных дисклинациях, которые создают большие локальные растягивающие напряжения. Для анализа условий зарождения и распространения такой микротрещины воспользуемся методом конфигурационной силы [28]. Для плоской деформации изотропного материала выражение для конфигурационной силы $f$, определяемой как величина упругой энергии, выделяющейся при продвижении трещины на единичный отрезок, имеет вид

$$
f(l)_{\varphi}=\frac{l}{8 D}\left(\chi\left(\bar{\sigma}_{\varphi \varphi}\right) \bar{\sigma}_{\varphi \varphi}^{2}+\bar{\sigma}_{r \varphi}^{2}\right),
$$

где $D=G /[2 \pi(1-v)], G-$ модуль сдвига, $l-$ длина трещины, $\varphi$ - полярный угол, задающий ориентацию рассматриваемой трещины (рис. $3, b), \bar{\sigma}_{\varphi \varphi}, \bar{\sigma}_{r \varphi}-$ средневзвешенные суммарные напряжения в окрестности трещины:

$$
\begin{gathered}
\bar{\sigma}_{\varphi \varphi}=\frac{2}{\pi l} \int_{0}^{l} \sigma_{\varphi \varphi}(r, \varphi) \sqrt{\frac{r}{l-r}} d r, \\
\bar{\sigma}_{r \varphi}=\frac{2}{\pi l} \int_{0}^{l} \sigma_{r \varphi}(r, \varphi) \sqrt{\frac{r}{l-r}} d r, \\
\chi\left(\bar{\sigma}_{\varphi \varphi}\right)= \begin{cases}1, & \bar{\sigma}_{\varphi \varphi} \geq 0, \\
0, & \bar{\sigma}_{\varphi \varphi}<0,\end{cases}
\end{gathered}
$$

где $\sigma_{\varphi \varphi}, \sigma_{r \varphi}-$ компоненты напряжения в полярной системе координат.

При этом равновесные длины трещин $l_{e q}$ для заданного направления $\varphi$ будут определяться из соотношения $f\left(l=l_{e q}\right)=2 \gamma$, где $\gamma-$ удельная энергия свободной поверхности. При этом длина трещины $l_{s t}$ называется устойчивой или стабильной, если она равновесная и выполняется условие $f_{l}^{\prime}\left(l=l_{e q}\right)<0$, а длина $l_{u n}$ называется неустойчивой или нестабильной, если она равновесная и выполняется условие $f_{l}^{\prime}\left(l=l_{e q}\right)>0$.

В работе [28] было показано, что в зависимости от типа расходимости функции напряжений вблизи сингулярного источника напряжений $\sigma \sim r^{-\alpha}$ могут реализоваться три характерных случая:

1) $\alpha=1 / 2$ : конфигурационная сила не зависит от длины трещины, при $f \geq 2 \gamma$ зарождение трещины и ее распространение происходит беспрепятственно;

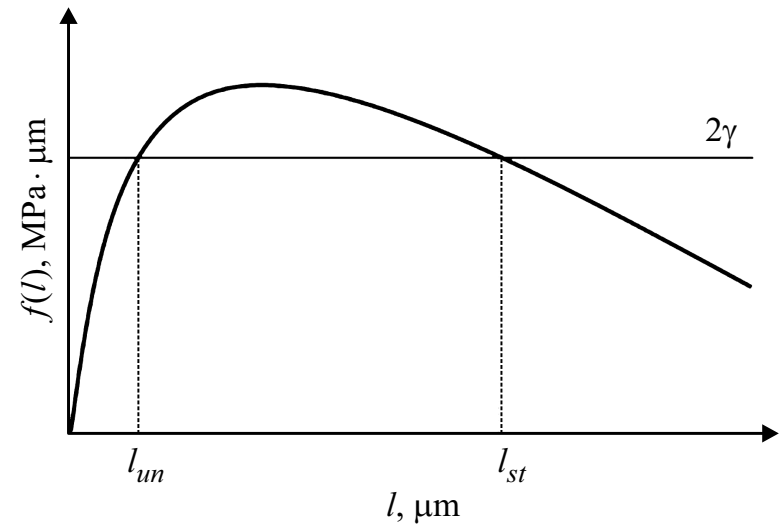

Рис. 4. Схематичное изображение зависимости конфигурационной силы $f$ от длины трещины $l$ для рассматриваемых мезодефектов при фиксированном $\varphi$.

2) $\alpha>1 / 2(f(0) \rightarrow \infty)$ : конфигурационная сила спадает по мере роста трещины, трещина достигает некоторой максимальной длины и останавливается;

3) $\alpha<1 / 2(f(0) \rightarrow 0)$ : конфигурационная сила растет по мере увеличения роста трещины, наиболее сложным этапом разрушения является зарождение трещины.

Для приведенных мезодефектов при малых $r$ наблюдается логарифмическая расходимость локальных полей напряжений [21], что соответствует третьему случаю. При этом характерная зависимость конфигурационной силы $f$ от длины трещины $l$ для рассматриваемых мезодефектов при фиксированном $\varphi$ имеет вид, приведенный на рис. 4.

Из рис. 4 следует, что конфигурационная сила $f<2 \gamma$ при $l_{*}<l_{u n}$ и подрастание трещины от нулевой до некоторой длины $l_{*}<l_{u n}$ не может происходить без дополнительной подкачки энергии (по определению конфигурационной силы). Вследствие чего процесс зарождения трещины в рассматриваемых полях упругих напряжений происходит за счет иных механизмов.

Для оценки концентрации трещин будем считать, что на частицах мартенсита уже имеются нанонесплошности и нанотрещины с характерным размером $l_{*}$, которые появились на этапе образования частицы. Будем считать, что функция распределения $l_{*}$ описывается экспоненциальным законом:

$$
F\left(l_{*}\right)= \begin{cases}0, & l_{*}<0 \\ 1-\exp \left(-\frac{1}{L} l_{*}\right), & l_{*} \geq 0\end{cases}
$$

где $F\left(l_{*}\right)$ - функция экспоненциального распределения, $L$ - подгоночный параметр.

Тогда на каждой частице с фиксированной ориентацией и при фиксированном значении величины $\varepsilon_{p l}$ имеется нанонесплошность (нанотрещина) с характерным размером (с длиной) $l_{*}$, распределенным согласно $F\left(l_{*}\right)$. Причем имеющиеся на частицах нанонесплошности смогут превратиться в стабильную трещину длины $l_{s t}$ только 
при выполнении следующего условия:

$$
l_{*} \geq l_{u n}^{\min }, \quad l_{u n}^{\min }=\min _{\varphi}\left[l_{u n}(\varphi)\right] .
$$

Отсюда следует, что будет раскрываться только некоторая часть нанонесплошностей. Концентрация частиц $n_{\mathrm{cr}}$, ориентированных вдоль направления $\Omega$ при фиксированной пластической деформации $\varepsilon_{p l}$, на которых появятся стабильные трещины, определяется с помощью следующего выражения:

$$
d n_{\mathrm{cr}}\left(\Omega, \varepsilon_{p l}\right)=d n_{m} P\left(l_{*} \geq l_{u n}^{\mathrm{min}}\right),
$$

где $P\left(l_{*} \geq l_{u n}^{\min }\right)-$ вероятность того, что длина нанонесплошности $l_{*}$ будет больше $l_{u n}^{\min }$ :

$$
P\left(l \geq l_{u n}^{\min }\right)=F( \pm \infty)-F\left(l_{u n}^{\min }\right)=\exp \left(-\frac{1}{L} l_{u n}^{\min }\right) .
$$

При этом средняя длина стабильных трещин $\bar{l}_{s t}$ определяется как

$$
\bar{l}_{s t}=\int_{l_{u n}^{\min }}^{\infty} l_{s t} P\left(l_{*} \mid l_{*} \geq l_{u n}^{\min }\right) d l,
$$

где $P\left(l_{*} \mid l_{*} \geq l_{u n}^{\min }\right)$ - условная плотность вероятности величины $l_{*}, l_{s t}$ - максимальная стабильная длина, соответствующая зародышевой трещины длины $l_{u n}$. Тогда поврежденность $d\left(\psi^{(t h)}\right)$, связанная с появлением стабильных трещин на частицах, ориентация которых лежит в интервале углов $[\Omega, \Omega+d \Omega]$ (с учетом симметрии), вычисляется следующим образом:

$$
d\left(\psi^{(t h)}\right)=d n_{\mathrm{cr}} \bar{l}_{s t}^{3} .
$$

Суммарная поврежденность материала $\psi^{(t h)}$ при фиксированной величине пластической деформации получается интегрированием по всему диапазону ориентаций частиц $\Omega$ (с учетом симметрии)

$$
\psi^{(t h)}=\int_{0}^{\pi / 2} d \psi^{(t h)}
$$

\section{4. Результаты численных расчетов}

Численные расчеты будем проводить при следующих значениях параметров: $G=78000 \mathrm{MPa}, v=0.294$, $2 a_{1}=1 \mu \mathrm{m}, \quad 2 a_{2}=0.2 \mu \mathrm{m}-$ размеры включения, $\varepsilon_{0}=0.02$ - соответствует тому, что объем мартенситной частицы увеличился на $2 \%$ [29]. Удельную поверхностную энергию трещины, образующейся в аустените или мартенсите, оценим, согласно [30], как $\gamma_{a}=G b_{a} / 10$ и $\gamma_{m}=G b_{m} / 10$ соответственно, $b_{a}=3.6 \cdot 10^{-4} \mu \mathrm{m}$ параметр решетки аустенита, $b_{m}=2.9 \cdot 10^{-4} \mu \mathrm{m}-$ параметр решетки мартенсита. Удельную поверхностную

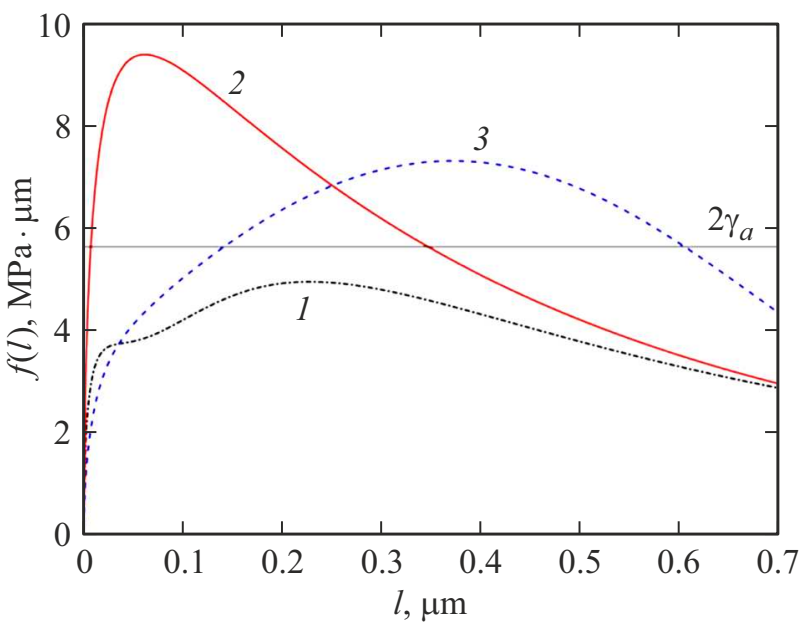

Рис. 5. Характерные зависимости конфигурационной силы $f$ от длины трещины $l$ при ее углах ориентациях $\varphi=10$ (1), 60 (2) и $140^{\circ}$ (3) и фиксированных значениях $\varepsilon_{p l}=0.08$ и $\Omega=\pi / 3$.

энергию трещины, образующейся на некогерентной межфазной границе, $\gamma_{b}$ определим так же, что и для большеугловой границы зерна, т.е. $\gamma_{b}=G b_{m} / 10-G b_{m} / 48$. Диапазон изменения величины пластической деформации $\varepsilon_{p l}=0.00-0.10$.

В результате расчетов получены характерные зависимости конфигурационной силы $f$ от длины трещины $l$ при разных значениях угла ориентации $\varphi$ и фиксированных значениях $\varepsilon_{p l}=0.08$ и $\Omega=\pi / 3$ (рис. 5). На рис. 5 в качестве примера приведены характерные зависимости $f$ от длины трещины $l$ при углах ориентациях $\varphi=10,60$ и $140^{\circ}$, соответствующих аустенитной матрице. Как видно, существование стабильной трещины возможно не при всех углах ориентациях $\varphi$, а только при тех, для которых выполняется условие $f_{\max }(l)>2 \gamma$. Для рассматриваемого случая это угол ориентации $\varphi=60$ и $140^{\circ}$. Помимо этого, значения длин равновесных трещин для разных углов ориентаций могут существенно отличаться. Так, например, для угла ориентации $\varphi=60^{\circ}$ длина зародышевой трещины на порядок меньше, чем для угла ориентации $\varphi=140^{\circ}$, что говорит о том, что вероятность появления стабильной трещины в первом направлении гораздо выше, чем во втором.

C помощью построения аналогичных зависимостей $f(l)$ для всего диапазона углов $\varphi \in[02 \pi)$ были определены длины трещин $l_{u}$ и $l_{s t}$. На рис. 6 приведены зависимости этих длин $l_{u n}$ (рис. 6,a) и $l_{s t}$ (рис. 6,b) от угла ориентации $\varphi$, полученные при фиксированных значениях $\varepsilon_{p l}=0.08$, и $\Omega=\pi / 3$. Из рис. $6, a$ видно, что зародышевые трещины $l_{u n}$ при фиксированных значениях $\varepsilon_{p l}=0.08$, и $\Omega=\pi / 3$ для диапазона углов $\varphi \in(\pi / 32 \pi / 3) \cup\{\pi\}$ принимают наименьшие значения, следовательно, появление стабильных трещин, длины которых приведены на рис. 6, $b$, 

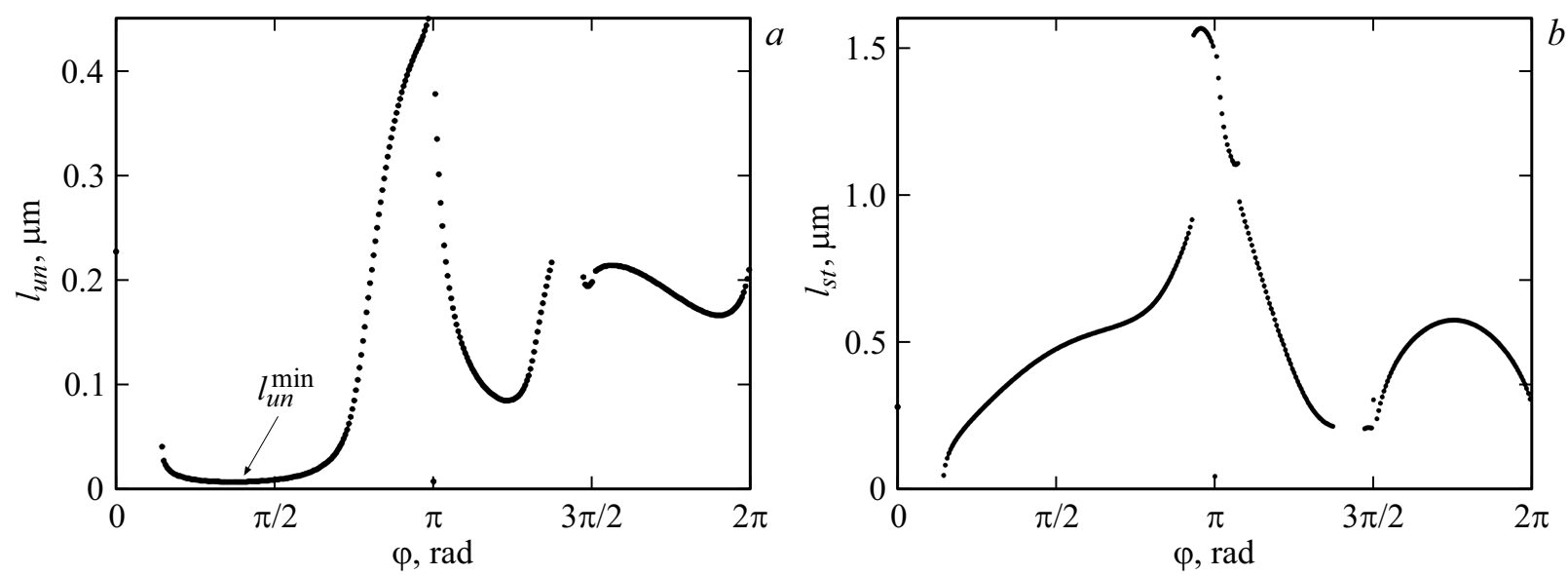

Рис. 6. Зависимости $l_{u n}(\varphi)(a)$ и $l_{s t}(\varphi)(b)$ при фиксированных значениях $\varepsilon_{p l}=0.08$, и $\Omega=\pi / 3$.

в этом диапазоне наиболее вероятно. В то время как для углов, соответствующих мартенситной частицы $\varphi \in(\pi 3 \pi / 2)$, зародышевые трещины достаточно большие, вследствие чего вероятность формирования стабильных трещин в частице мартенсита пренебрежимо мала.

Далее, определяя зависимости $l_{u n}(\varphi)$ и $l_{s t}(\varphi)$ для всех значений величин $\Omega$ (шаг по углу $\Delta \Omega=10^{\circ}$ ) и всех значений $\varepsilon_{p l}$ (шаг по деформации $\left.\Delta \varepsilon_{p l}=0.01\right)$, получим зависимость $\psi\left(\varepsilon_{p l}\right)$ и, согласно выражению (3), $\Delta v^{(t h)}$. Значение подгоночного параметра $L$ определим из условия минимальности отклонения теоретической зависимости $\Delta v_{\psi}^{(t h)}\left(\varepsilon_{p l}\right)$ от экспериментальной зависимости $\Delta v_{\psi}^{(\exp )}\left(\varepsilon_{p l}\right)$ :

$$
\sum\left|\Delta v_{\psi}^{(t h)}\left(\varepsilon_{p l}\right)-\Delta v_{\psi}^{(\exp )}\left(\varepsilon_{p l}\right)\right| \rightarrow \min
$$

Данное условие выполняется при $L=174 b_{a} \cong$ $\cong 0.0626 \mu \mathrm{m}$.

Таким образом, для того чтобы получить оценочные значения поврежденности, (выражение (12)) и изменение коэффициента Пуассона $\Delta v_{\psi}$ (выражение (3)), необходимо задать геометрию и распределение ориентаций мартенситных частиц, определить концентрацию частиц мартенсита (выражение (6)), несовместность пластической деформации (выражение (7)), дилатации (выражение (8)), и рассчитать поля упругих напряжений от частиц мартенсита (П1-П6). Далее с помощью метода конфигурационной силы (выражение (9)) определить длины равновесных (стабильных и нестабильных) трещин, формирующихся в полях упругих напряжений от частиц мартенсита. Для определения концентрации трещин и их средней длины по формулам (10) и (11) соответственно использовать подгоночный параметр, полученный на основе экспериментальных данных по формуле (13).

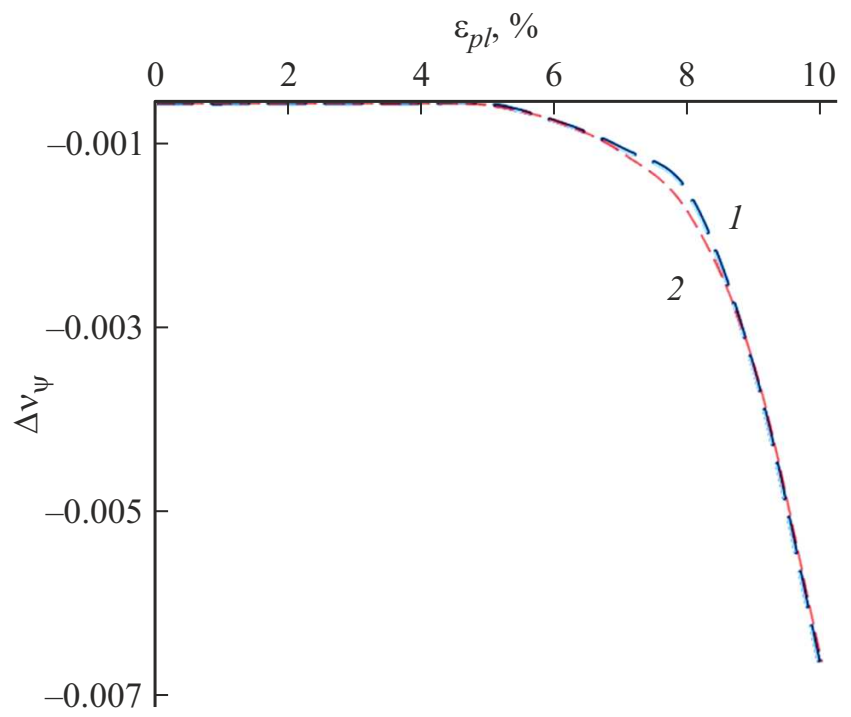

Рис. 7. Сравнение $v_{\psi}^{(\exp )}(1)$ и $v_{\psi}^{(t h)}(2)$ на ранних стадиях пластического нагружения.

\section{5. Обсуждение результатов}

Изменения коэффициента Пуассона $\Delta v_{\psi}$ в процессе пластического деформирования, полученные экспериментально (формула (5)) и теоретически, представлены на рис. 7.

Наблюдается хорошая корреляция между теоретическими расчетными и экспериментальными данными при деформациях $\varepsilon_{p l}$, не превышающих 10\%.

Проведенные расчеты подтвердили, что поврежденность не оказывает влияние на коэффициент Пуассона $v_{\psi}$ при деформациях $\varepsilon_{p l}$, не превышающих $5 \%$. При дальнейшем деформировании на частицах деформационного мартенсита появляются новые системы ротационно-сдвиговых мезодефектов, которые создают внутренние напряжения, в полях которых образуются микротрещины с определенной ориентацией. Новые де- 
фекты увеличивают составляющую $v_{\psi}$ по абсолютному значению.

\section{Заключение}

Исследовано влияние пластического деформирования на коэффициент Пуассона, измеренного акустическим методом, и интенсивность образования деформационного мартенсита, величина которого получена с помощью вихретоковых измерений. Экспериментальные исследования показали, что основное влияние на изменение коэффициента Пуассона оказывают связанные друг с другом процессы: образование деформационного мартенсита и накопление микроповрежденности (микропор, микротрещин).

Получено, что активное изменение коэффициента Пуассона сопровождается активным ростом деформационного мартенсита. На поздних стадиях деформирования интенсивность изменения уменьшается, предположительно из-за образования микроповрежденности.

Проведенное моделирование формирования стабильных трещин, зарождающихся вблизи ротационносдвиговых мезодефектов, на границе включение-матрица показало, что на начальном этапе, учитывая малый размер трещин, вклад в приращение коэффициента Пуассона за счет увеличения объемной доли деформационного мартенсита превалирует над вкладом за счет увеличения количества трещин. Получено, что плотность стабильных микротрещин на частицах деформационного мартенсита растет как за счет увеличения количества самих микротрещин, так и за счет прорастания уже имеющихся микротрещин. Этот процесс изменяет монотонный ход зависимости величины коэффициента Пуассона от пластической деформации. Проведенное сравнение теоретических и экспериментальных результатов показало хорошее совпадение кривых при малых степенях деформирования, что может говорить о правомерности выбранного рассматриваемой в работе теоретической модели.

\section{Финансирование работы}

Работа выполнена при финансовой поддержке Российского научного фонда в рамках гранта № 19-19-00637.

\section{Конфликт интересов}

Авторы заявляют, что у них нет конфликта интересов.

\section{Приложение}

Выражения для расчета компонентов тензора полей упругих напряжений мезодефектов [22,23], определенных в правосторонней декартовой системе координат $O x y$, начало которой совпадает с центром мезодефекта, а ось $O x$ направлена вдоль плеча мезодефекта, имеют вид
Планарный сдвиговый мезодефект:

$$
\begin{aligned}
\sigma_{x x}= & D w_{\tau}\left[2\left(\operatorname{arctg}\left[\frac{x-a}{y}\right]-\operatorname{arctg}\left[\frac{x+a}{y}\right]\right)\right. \\
& \left.+\frac{y(x+a)}{(x+a)^{2}+y^{2}}-\frac{y(x-a)}{(x-a)^{2}+y^{2}}\right], \\
\sigma_{y y}= & D w_{\tau} y\left(\frac{(x-a)}{(x-a)^{2}+y^{2}}-\frac{(x+a)}{(x+a)^{2}+y^{2}}\right), \\
\sigma_{x y}= & D w_{\tau}\left(\frac{y^{2}}{(x+a)^{2}+y^{2}}-\frac{y^{2}}{(x-a)^{2}+y^{2}}\right. \\
& \left.+\frac{1}{2} \ln \left[\frac{(x+a)^{2}+y^{2}}{(x-a)^{2}+y^{2}}\right]\right) .
\end{aligned}
$$

Диполь клиновых дисклинаций:

$$
\begin{aligned}
& \sigma_{x x}= D w_{N}\left(\frac{y^{2}}{(x+a)^{2}+y^{2}}-\frac{y^{2}}{(x-a)^{2}+y^{2}}\right. \\
&\left.+\frac{1}{2} \ln \left[\frac{(x+a)^{2}+y^{2}}{(x-a)^{2}+y^{2}}\right]\right), \\
& \sigma_{y y}= D w_{N}\left(\frac{y^{2}}{(x-a)^{2}+y^{2}}-\frac{y^{2}}{(x+a)^{2}+y^{2}}\right. \\
&\left.+\frac{1}{2} \ln \left[\frac{(x+a)^{2}+y^{2}}{(x-a)^{2}+y^{2}}\right]\right), \\
& \sigma_{x y}=D w_{N}\left(\frac{(x-a) y}{(x-a)^{2}+y^{2}}-\frac{(x+a) y}{(x+a)^{2}+y^{2}}\right),
\end{aligned}
$$

где $w_{N}, w_{\tau}$ - величины проекций мощностей мезодефектов $\mathbf{w}_{\mathbf{N}}, \mathbf{w} \boldsymbol{\tau}$ на ось $O x, 2 a$ - длина сдвигового мезодефекта или дисклинационного диполя.

\section{Список литературы}

[1] В.В. Мишакин, В.А. Клюшников, А.В. Гончар. ЖТФ, 85 (5), 32 (2015). [V.V. Mishakin, V.A. Klyushnikov, A.V. Gonchar. Tech. Phys., 60 (5), 665 (2015). DOI: $10.1134 / \mathrm{S} 1063784215050163]$

[2] А.В. Гончар, В.В. Мишакин, В.А. Клюшников, К.В. Курашкин. ЖТФ, 87 (4), 518 (2017). DOI: $10.21883 /$ JTF.2017.04.44310.1828 [A.V. Gonchar, V.V. Mishakin, V.A. Klyushnikov, K.V. Kurashkin. Tech. Phys., 62 (4), 537 (2017). DOI: 10.1134/S1063784217040089]

[3] A.V. Gonchar, V.V. Mishakin, V.A. Klyushnikov. Int. J Fatigue., 106, 153 (2018). DOI: 10.1016/j.ijfatigue.2017.10.003

[4] V. Mishakin, A. Gonchar, K. Kurashkin, M. Kachanov. Int. J Fatigue., 141, 105846 (2020). DOI: $10.1016 /$ j.jfatigue.2020.105846

[5] V. Mishakin, A. Gonchar, K. Kurashkin, V. Klyushnikov, M. Kachanov. Int. J. Eng. Sci., 168, 103567 (2021). DOI: $10.1016 / j . i j e n g s c i .2021 .103567$ 
[6] В.Ф. Терентьев, А.Г. Колмаков, В.М. Блинов. Деформация и разрушение материалов, 6, 2 (2007).

[7] А.С. Вавакин, Р.Л. Салганик. Механика твердого тела, 3, 65 (1975).

[8] Р.Л. Салганик, Механика твердого тела. 4, 149 (1973).

[9] J.R. Bristow. Brit. J. Appl. Phys., 11 (2), 81 (1960). DOI: $10.1088 / 0508-3443 / 11 / 2 / 309$

[10] M. Kachanov, I. Sevostianov. Micromechanics of Materials, with Applications (Springer, Cham. 2018), DOI: $10.1007 / 978-3-319-76204-3$

[11] E.A. Soppa, C. Kohler, E. Roos. Mat. Sci. Eng. A, 597, 128 (2014). DOI: 10.1016/j.msea.2013.12.036

[12] A.A. Zisman, V.V. Rybin. Acta Mater., 44, 403 (1996). DOI: $10.1016 / 1359-6454(95) 00155-8$

[13] V.N. Perevezentsev, G.F. Sarafanov. Rev. Adv. Mater. Sci., 30 (1), 73 (2012).

[14] A.E. Romanov, A.L. Kolesnikova. Progr. Mater. Sci., 54, 740 (2009). DOI: 10.1016/j.pmatsci.2009.03.002

[15] G.B. Olson, M. Cohen. Metall Trans. A, 6A, 791 (1975).

[16] J. Post, H. Nolles, K. Datta, H.J.M. Geijselaers. Mat. Sci. Eng. A, 498 (1-2), 179 (2008). DOI: 10.1016/j.msea.2008.07.051

[17] F. Kroupa, L. Lejcek. Czech. J. Phys., 20, 1063 (1970).

[18] I.A. Ovid'ko, A.G. Sheinerman. Rev. Adv. Mater. Sci., 9 (1), 17 (2005).

[19] J.F. Nye. Acta Met., 1, 153 (1953).

[20] R. Bullough, B.A. Bilby. Proc. Roy. Soc., B69, 1276 (1956).

[21] С.В. Кириков, А.С. Пупынин, Ю.В. Свирина. Проблемы прочности и пластичности, 83 (2), 235 (2021). DOI: $10.32326 / 1814-9146-2021-83-2-235-244$

[22] В.А. Лихачев, Р.Ю. Хайров. Введение в теорию дисклинаций (ЛГУ, Л., 1975)

[23] С.В. Кириков, В.Н. Перевезенцев. Письма о материалах, 11 (1), 50 (2021). DOI: 10.22226/2410-3535-2021-1-50-54 [S.V. Kirikov, V.N. Perevezentsev. Lett. Mater., 11 (1), 50 (2021). DOI: 10.22226/2410-3535-2021-1-50-54]

[24] M.S. Wu. Int. J. Plasticity, 142, 100 (2018). DOI: $10.1016 /$ j.jplas.2017.10.001

[25] Г.Ф. Сарафанов, В.Н. Перевезенцев. Деформация и разрушения материалов, 2, 2 (2016).

[26] М.Ю. Гуткин, И.А. Овидько, Н.В. Скиба. ФТТ, 49 (2), 252 (2007). [M.Yu. Gutkin, I.A. Ovid'ko, N.V. Skiba. Phys. Solid State, 49, 261 (2007). DOI: 10.1134/S1063783407020138]

[27] M.Yu. Gutkin, I.A. Ovid'ko. Phil. Mag. Lett., 84 (10), 655 (2004). DOI: $10.1080 / 09500830512331329123$

[28] В.Л. Инденбом. ФТТ, 3, 2071 (1961).

[29] В.Г. Чащина, М.П. Кащенко. Экспериментальные основания динамической теории мартенситных превращений (Изд-во Урал. ун-та, Екатеринбург, 2020)

[30] J. Friedel. Dislocations (Pergamon Press, Oxford, 1964) 